\title{
Atomabsorptionsspektrometrische Bestimmung von Blei in Blut und Harn mit dem Delves-Sampling-System
}

\author{
Von G. HeinemanN \\ Aus dem Institut für Klinische Cbemie und Klinische Biochemie (Direktor: Prof. Dr. H.-J. Dulce) \\ am Klinikum Steglitz der Freien Universität Berlin
}

(Eingegangen am 15. Januar/20. Februar 1973)

Blei kann im heparinisierten Vollblut und im Nativharn quantitativ mit Hilfe der Probenbecher-Technik nach Decves atomabsorptionsspektrometrisch bestimmt werden. Die benötigte Probenmenge beträgt lediglich $10 \mu \mathrm{l}$ und wird nach einer teilweisen Oxidation mit Wasserstoffperoxid und anschließender Trocknung analysiert. Eine Extraktion mit einem organischen Lösungsmittel nach vorangegangener Chelatbildung entfällt. Die hohe Empfindlichkeit des Verfahrens gestattet Bleimengen bis 0,1 ng zu messen. Die Methode ist einfach, zuverlässig und erfordert geringen Zeitaufwand. Für eine einzelne Blutbleibestimmung werden weniger als 5 min benötigt. Bei Serienmessungen lassen sich 30-40 Proben pro Stunde aufgrund der kurzen Probenvorbereitungszeit bestimmen. Damit dürfte dieses Verfahren für Reihenuntersuchungen bleiexponierter Personen an Bedeutung gewinnen.

\section{Determination of lead in blood and urine by atomic absorption spectrometry using the DeLves sampling system}

Lead can be determined quantitatively in heparinized whole blood and in untreated urine by atomic absorption spectrometry, using the sampling technique of DeLVEs. Only $10 \mu \mathrm{l}$ of sample are required; this is partially oxidized with hydrogen peroxide and taken to dryness prior to analysis. In this procedure, chelate formation followed by extraction with organic solvent is omitted. The sensitivity is sufficient to measure $0.1 \mathrm{ng}$ of lead. The method is simple, reliable and quick. A single blood lead determination takes less than $5 \mathrm{~min}$. Owing to the short preparation time for the sample, 30-40 samples can be determined serially in one hour. This could therefore become an important method for the serial investigation of persons exposed to lead.

Die Bestimmung von Blei im Blut und im Harn ist seit langem möglich und hat für die arbeitsmedizinische Betreuung von Personen, die in der bleiverarbeitenden Industrie einer chronischen Bleieinwirkung ausgesetzt sind, und bei der Diagnostik und Therapie akuter Bleiintoxikationen besondere Bedeutung erlangt. Allerdings zählen bisher Bleibestimmungen in Blut noch immer zu den Analysenverfahren, die aufwendig und zeitraubend sind, so $\mathrm{da} ß$ sie routinemäßig nur an wenigen Instituten durchgeführt werden.

Die Methoden zur Bestimmung von Blei in Blut oder Harn teichen von kolorimetrischen Verfahren unter Anwendung von Dithizon über die Emissions-Spektroskopie bis zur Atom-Absorptions-Spektrometrie, die sich durch Spezifität und hohe Empfindlichkeit auszeichnet und weitgehend frei von Störungen durch Lösungspartner ist. Eine Ubersicht weiterer Methoden zur Bleibestimmung unter Hinweis auf ihre Vor- und Nachteile geben WACHTER und SALLABERger (1) sowie Christian (2).

Als Methode der Wahl für die Bestimmung von Blei in Blut oder Harn gilt zur Zeit die Atom-AbsorptionsSpektrometrie. Die konventionelle Methode der atomabsorptionsspektrometrischen Bestimmung von Blei in Blut, die von Berman (3), Willis (4), Sprague und SLAviN (5) und zunächst auch von LEHNERT und SCHALLER (6) angewendet wurde, beinhaltet eine einfache, jedoch zeitaufwendige Probenvorbereitung. Nach
Enteiweißung der Probe, Einstellen des pH-Wertes, Zugabe eines Komplexbildners und Überführung des Bleichelats in ein organisches Lösungsmittel wurde dieses in die Flamme gesprüht. Auf diese Weise konnte nicht nur eine Anreicherung des Metalls in der organischen Phase, sondern auch eine etwa fünffache Empfindlichkeitssteigerung erzielt werden. Später wurde von LEHNERT et al. (7) eine Vereinfachung dieser Methode angegeben, wobei die Enteiweißung der Probe entfällt. Die Probenmenge konnte reduziert und der gesamte Arbeitsgang in einem Gefäß durchgeführt werden. Auch wir bestimmten anfangs Blei im Blut nach dieser Methode, verwendeten jedoch zur Zerstörung der Erythrocyten ein Ultraschallgerät.

Seit einiger Zeit benutzen wir eine Variante der AtomAbsorptions-Spektrometrie, die Probenbecher-Technik von Delves (8), die gegenüber der Boot-Technik von KAHN et al. (9) weitere Vorteile bietet. Beide Varianten wie auch das Verfahren der Graphitrohrküvette, über das Matousek und Strevens (10) berichten, zählen zu den Mikroprobentechniken. Es werden Probenmengen zwischen $10 \mu \mathrm{l}(8)$ und $20 \mu \mathrm{l}(9,10)$ Blut benötigt.

Die Arbeit hat den Zweck, die neue Methode zu erläutern, ihre Anwendbarkeit, Störanfälligkeit und Zuverlässigkeit zu prüfen, sowie Grenzwerte für die Beurteilung des mit dieser Methode ermittelten Bleigehalts in Blut oder Harn anzugeben. 


\section{Methodik}

\section{Prinzip der Probenbechertechnik}

Die Bleibestimmung mit Hilfe des Zusatzes für Substanzdirektverdampfung basiert auf dem Prinzip der Atom-AbsorptionsSpektrometrie. Bezüglich näherer Angaben über dieses Verfahten sei auf die Arbeiten von Allan (11), HerrmanN (12), Willis (13), Paschen und Spiekermann (14) und Lehnert (15), der Hinweise speziell für Bleibestimmungen gibt, hingewiesen.

Hier soll näher auf das Probenbecher-System eingegangen werden, das schematisch in Abbildung 1 wiedergegeben ist. Uber einem Longitudinalbrenner (Boling-Dreischlitzbrenner) wird das sogenannte Absorptions-Rohr auf zwei Sockeln befestigt. Die Abmessungen des Rohrs, das aus dünnem Nickelblech besteht, betragen $10 \mathrm{~cm}$ in der Länge und $1,25 \mathrm{~cm}$ im Durchmesser. Das Rohr ist beiderseits offen und trägt im Boden eine weitere Offnung für den Eintritt der atomisierten Probe. Das Rohr ist so zu justieren, daß die Pb-Resonanzlinie der Blei-Lampe das Rohr zentral passiert. Der Probenbecher hängt in einer Platin-Iridium-Drahtschlinge und wird zum Messen der Probe in die Flamme geschoben. Er sollte sich etwa $2 \mathrm{~mm}$ unterhalb der Öffnung im Boden des Rohrs befinden, so daß der Atomdampf an dieser Stelle in das Rohr eintreten kann. Die Absorption findet innerhalb dieses Rohrs statt.

Bei dieser atomabsorptionsspektrometrischen Variante wird Blei quantitativ bestimmt. Die Ermittlung des Endwerts erfolgt entweder graphisch nach Anwendung des Additionsverfahrens bei der Probenvorbereitung oder aber rechnerisch mit Hilfe eines selbsthergestellten :Sțandards.

\section{Reagenzien}

Ammoniumpyrrolidindithiocarbamat, Fa. Heyl \& Co., Berlin Methylisobutylketon zur Extraktionsanalyse (Merck),

Perhydrol p. a., $300 \mathrm{~g} / \mathrm{kg}$ (Merck),

Blei(II)-nitrat p. a. (Merck).

\section{Lösungen}

1. Bleistammlösung $1 \mathrm{mmol} / 1: 331,20 \mathrm{mg}$ Blei(II)-nitrat werden in einen 1 Liter-Meßkolben gegeben. Mit tridest. Wasser wird bis zur Marke aufgefüllt. Diese Stammlösung enthält $1 \mathrm{mmol} / \mathrm{l}$ Blei und wird in einer Polyäthylenflasche aufbewahrt.

2. Bleilösung $100 \mu \mathrm{mol} / 1: 10 \mathrm{ml}$ der Bleistammlösung werden in einen 100 ml-Meßkolben pipettiert und mit tridest. Wasser bis zur Marke aufgefüllt. Die Lösung sollte öfters neu angesetzt werden.

3. Eichlösungen 2 und $4 \mu \mathrm{mol} / 1$ Blei: $2 \mathrm{ml} \mathrm{bzw.} 4 \mathrm{ml}$ der Bleilösung $100 \mu \mathrm{mol} / 1$ werden in einen $100 \mathrm{ml}-\mathrm{Me} ß k$ kolben pipettiert und mit tridest. Wasser bis zur Marke aufgefüllt. Eichlösungen stets neu ansetzen.

4. Ammoniumpyrrolidindithiocarbamatlösung $50 \mathrm{~g} / \mathrm{l}$.

Geräte

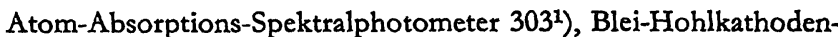
lampe ${ }^{\mathrm{x}}$ ), Hitachi-Schreiber Modell 165), Zusatz für Substanzdirektverdampfung ${ }^{1}$ ), Probentiegel ${ }^{1}$ ), Eppendorf-Gefäße, Eppen-

1) Bodenseewerk Perkin-Elmer, Uberlingen.

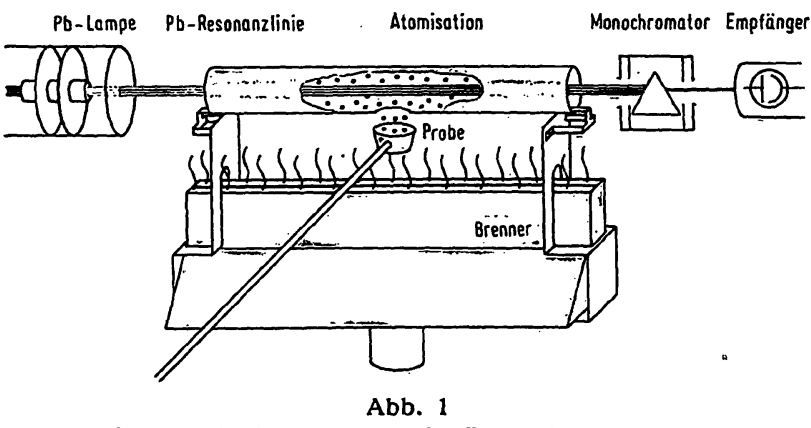

Schema der Verdampfung im Probenbechersystem
dorf-Pipetten $10 \mu \mathrm{l}$ und $20 \mu \mathrm{l}$, thermostatisierte Heizplatte (einstellbar auf $140^{\circ} \mathrm{C}$.

\section{Untersuchungsgang}

\section{Additionsverfabren}

Heparinisiertes Vollblut oder Nativharn wird zunächst in ein Eppendorfgefäß gefüllt. Je $10 \mu \mathrm{l}$ Probe werden in 6 Tiegel pipettiert. In den 3. und 4. Tiegel werden je $10 \mu \mathrm{l}$ der Blei-Eichlösung $2 \mu \mathrm{mol} / \mathrm{l}$, in Tiegel 5 und $610 \mu \mathrm{l}$ der Blei-Eichlösung $4 \mu \mathrm{mol} / 1$ zugegeben. Danach erfolgt die exste Trocknung der Proben bei $140^{\circ} \mathrm{C}$, die etwa $30-60 \mathrm{~s}$ dauert. Anschließend nimmt man die Proben von der Heizplatte, läßt abkühlen und gibt je $20 \mu \mathrm{l} \mathrm{H}_{2} \mathrm{O}_{2} 300 \mathrm{~g} / \mathrm{kg}$ in alle 6 Tiegel. Für Harnbleibestimmungen ist die $\mathrm{Zugabe}$ von $\mathrm{H}_{2} \mathrm{O}_{2}$ im allgemeinen nicht notwendig. Die Proben werden nochmals bei $140^{\circ} \mathrm{C}$ etwa 2 min getrocknet. Hierbei fällt die zunächst schaumige Blutprobe im Tiegel zusammen und verkrustet. Die Probe ist nun fertig zur Messung. Die Temperatur von $140^{\circ} \pm 5^{\circ} \mathrm{C}$ läßt sich mit einer thermostatisierten Heizplatte konstant halten und sollte nicht erhöht werden, da Perhydrol bei $150^{\circ} \mathrm{C}$ entzündbar ist.

\section{Mit Hilfe eines Standards}

Für Blutbleimessungen wird wegen des Matrixeffekts gleichfalls Blut, dem eịe wäßrige Blei-Eichlösung zugesetzt wird, als Standard verwendet. Die Konzentration des Standards wird zunächst nach dem Additionsverfahren ermittelt, danách werden die Blutproben in Doppelbestimmungen gemessen.

\section{Messung}

Der Meßvorgang beginnt mit dem Einbringen des Probentiegels in die Flamme. Atomisation und Absorption verlaufen im Anschluß daran innerhalb kürzester Zeit. Als Zeichen einer optimalen Absorption werden vom Schreiber scharfe Peaks in typischer Folge registriert. Voraussetzung ist eine optimale Geräteeinstellung, für die Richtwerte in Tabelle 1 angegeben sind.

\section{Schreibereichung}

Bei vollem Lichtdurchgang wird die Schreibernadel mit dem Knopf „Full Scale“ am Steuergetät auf $0 \%$ Absorption gebracht. Die Einstellung der Nadel auf 100\% Absorption mit Knopf "Zero" am Schreiber exfolgt durch Einbringen einer Blende in den Lichtstrahl der Blei-Lampe zwischen Flamme und Monochromator.

\section{Kurvenauswertung}

In Abbildung 2 sind Peaks einer Bleibestimmung in Blut und Harn wiedergegeben, wie sie bei Anwendung des Additionsverfahrens zur Darstellung kommen. Typisch für die Kurven bei

Tab. 1

Geräteinstellung für das Atom-Absorptions-Spektralphotometer mit angeschlossenem Schreiber zur Bestimmung von Blei in Blut und Harn

\begin{tabular}{lcc}
\hline \multicolumn{1}{c}{ Atom-Absorptions-Spektrometer } & \multicolumn{1}{c}{$\begin{array}{c}\text { Hitachi- } \\
\text { Schreiber }\end{array}$} \\
\hline Wellenlänge nm & 283,3 & $\begin{array}{l}\text { Papiergeschwindigkeit: } \\
60 \mathrm{~mm} / \mathrm{min}\end{array}$ \\
$\begin{array}{l}\text { Spaltbreite } \\
\text { Lampenstrom mA }\end{array}$ & 4 & $\begin{array}{l}\text { Spannungsschalter: } \\
10 \mathrm{mV}\end{array}$ \\
$\begin{array}{l}\text { Dämpfung } \\
\text { Skalendehnung } \\
\text { Brenner }\end{array}$ & $\begin{array}{l}\text { keine } \\
\text { keine }\end{array}$ & $\begin{array}{l}\text { Boling-Brenner mit } \\
\text { aufmontiertem Ab- }\end{array}$ \\
Brennerhöhe & $\begin{array}{l}\text { sorptionsrohr } \\
\text { Einstellung nach } \\
\text { optimalem Strahlen- } \\
\text { verlauf durch das } \\
\text { Absorptionsrohr }\end{array}$ \\
\hline
\end{tabular}




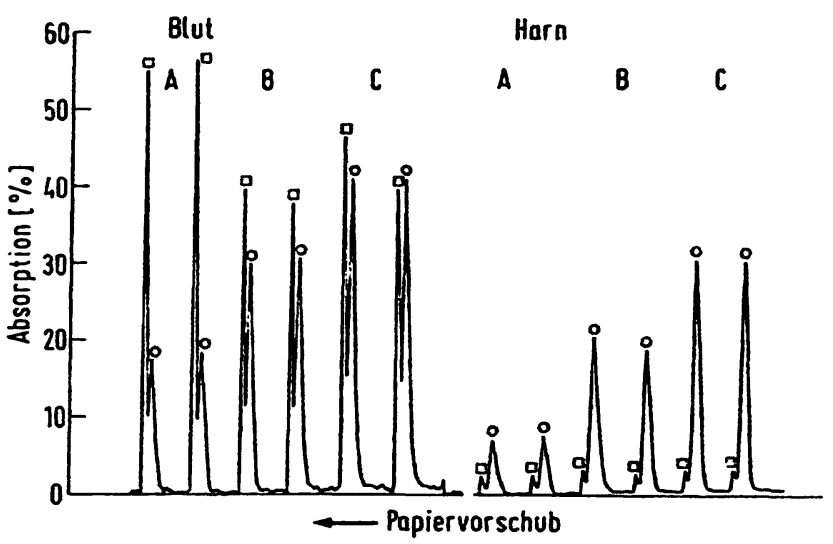

Abb. 2

Charakteristische Peaks bei der Bleibestimmung im Blut und Harn. Bleipeak $=0$, Rauchpeak $=\square, A$ : Probe ohne Pb-Zusatz, B Probe $+2 \mu \mathrm{mol} / 1$ Pb, C: Probe $+4 \mu \mathrm{mol} / 1$ Pb. A, B und C sind
Doppelbestimmungen. Papiergeschwindigkeit: $60 \mathrm{~mm} / \mathrm{min}$. Wellenlänge der MeBstrahlung $\lambda=283,3 \mathrm{~nm}$

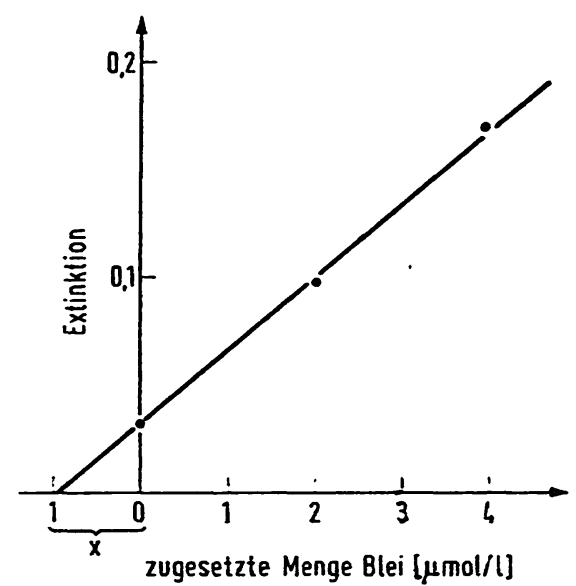

Abb. 3

Graphische Auswertung der bleispezifischen Peaks (vgl. Abb. 2) Extinktion bei einer Wellenlänge des MeBstrahls von $\lambda=283,3 \mathrm{~nm}$ $\mathbf{x}=$ Bleigehalt der Probe

Blutbleibestimmungen ist ihr Doppelgipfel, der aus dem stets vorangehenden Rauchpeak und dem bleispezifischen Peak besteht. Der Doppelgipfel erklärt sich aus der kurzfristigen rauchbedingten Lichtschwächung und der unmittelbar folgenden spezifischen Absorption. Gelegentlich kann sich ein weiterer unspezifischer kleiner Peak vor dem Rauchpeak zeigen. Die Rauchpeaks von meist unterschiedlicher Höhe resultieren aus der Verbrennung organischer Probenbestandteile. Bei Bleibestimmungen im Harn werden keine oder nur sehr kleine unspezifische Peaks erhalten. Entscheidend für die Auswertbarkeit der Kurven ist einzig eine exakte Trennung der Bleipeak-Spitze vom Rauchpeak, wobei die Höhe des Bleipeaks der prozentualen Absorption entspricht. Überlagerungen des Rauch- und Bleipeaks in den unteren Kurvenbereichen sind daher bedeutungslos. Bei ungenügender Trennung der Peaks ist das Wellenlängenoptimum zu überprüfen und eventuell neu einzustellen. Die graphische Auswertung und Ermittlung der gesuchten Konzentration zeigt Abbildung 3. Bei ungleicher Höhe der bleispezifischen Peaks ist der Mittelwert der Doppelbestimmungen zu nehmen. Da der benutzte Hitachi-Schreiber Absorptionswerte registriert, wie Abbildung 2 zeigt, werden diese mit Hilfe einer Tabelle in Extinktionen umgewandelt. Bei Messung eines Standards und einer bzw. mehrerer Proben werden gleichfalls Doppelbestimmungen durchgeführt. Die Extinktionen von Standard und Probe werden mit der Konzentration des Standards in Beziehung gesetzt und ergeben den Endwert nach der Gleichung

$$
C_{P}=\frac{E_{P}}{E_{S}} \times C_{S}
$$

$C_{P}=$ Konzentration der Probe, $C_{S}=$ Konzentration des Standards, $E_{P}=$ mittlere Extinktion der Probe, $E_{S}=$ mittlere Extinktion des Standards.

\section{Ergebnisse}

Linearitāt

Zur Prüfung der Linearität wurde Vollblut mit wäßrigen Bleilösungen steigender Konzentration versetzt, in der angegebenen Weise vorbereitet und gemessen. Die Eichkurve war linear bis $8,5 \mu \mathrm{mol} / \mathrm{l}$. Bei höheren Konzentrationen zeigte sich eine allmähliche Kurvendepression, so daß eine Probenverdünnung notwendig wurde. Für bleihaltige Harne fand sich eine Linearität bis mindestens $6 \mu \mathrm{mol} / 1 \mathrm{Blei}$, eine Konzentration, die auch bei Therapie mit Chelatbildnern nicht erreicht wird.

\section{Wiederfindung}

Blut

Zur Prüfung der Richtigkeit der Methode wurden 25 Blutproben (Gebalt an Blei $2,3 \mu \mathrm{mol} / \mathrm{l}$ ) mit $2 \mu \mathrm{mol} / 1$ Blei versetzt. Die Wiederauffindungsrate betrug zwischen 89,5 und $111 \%$, im Mittel 100,7\%. Für 24 Blutproben mit einem Gesamtbleigehalt von $2,40 \mu \mathrm{mol} / 1$ ergab sich eine Wiederfindung zwischen 90,0 und $109,0 \%$, im Mittel 98,0\%.

\section{Harn}

Es sollte geprüft werden, ob bei diesem Verfahren Harnsubstanzen einen Einfluß auf die bleispezifische Absorption auszuüben vermögen. Harnproben nicht bleiexponierter Personen wurden gemischt. Zur Entfernung möglicher Bleispuren wurden $50 \mathrm{ml}$ Mischharn mit $25 \mathrm{ml}$ Ammoniumpyrrolidindithiocarbamatlösung $50 \mathrm{~g} / 1$ versetzt und wenige Minuten geschüttelt. Anschließend wurden $25 \mathrm{ml}$ Methylisobutylketon dazugegeben, nochmals einige Minuten geschüttelt und die organische Phase abgesaugt. Den wäßrigen Eichlösungen mit einem Bleigehalt von 1, 2, 3, 4, 5 und $6 \mu \mathrm{mol} / \mathrm{l}$ wurde lediglich Methylisobutylketon $(2 \mathrm{ml}$ Eichlösung $+1 \mathrm{ml}$ Methylisobutylketon) zugegeben, geschüttelt und die organische Phase abgetrennt. Die so behandelten Eichlösungen wurden allein sowie zu gleichen Teilen mit Harn gemischt gemessen. Die erhaltenen Wertepaare gruppieren sich eng um eine Gerade mit der Gleichung $y=-0,05 x+1,01$. Der Korrelationskoeffizient war $r=0,995$. Die Wiederauffindungsrate der Harnbleibestimmungen für die 6 Eichpunkte betrug zwischen 92,0 und $106,2 \%$, im Mittel 99,5\%.

\section{Präzision}

Zur Bestimmung der Präzision in Serie wurden eine Blutprobe $24 \mathrm{mal}$ und eine Harnprobe $16 \mathrm{mal}$ gemessen. Bei einer mittleren Bleikonzentration im Blut von $2,35 \mu \mathrm{mol} / 1$ mit einer Standardabweichung von $\pm 0,124 \mu \mathrm{mol} / 1$ betrug der Variationskoeffizient $5,3 \%$. Im Harn fand sich eine mittlere Bleikonzentration von $1,08 \mu \mathrm{mol} / 1$ mit einer Standardabweichung 
von $\pm 0,059 \mu \mathrm{mol} / \mathrm{l}$. Der Variationskoeffizient betrug $5,43 \%$. Die Präzision von Tag zu Tag ergab für Blut $(n=14)$ einen Variationskoeffizienten von $3,27 \%$ und für Harn $(n=16) 4,23 \%$.

Bleigehalt im Blut und Harn von beruflich nicht bleibelasteten Personen

Die Blutbleibestimmung von 30 gesunden Personen ohne verstärkte Bleibelastung ergab einen mittleren Wert von $0,95 \pm 0,30 \mu \mathrm{mol} / \mathrm{l}(\overline{\mathrm{x}} \pm \mathrm{s})$ Blei. Im Harn von 20 Personen fand sich eine Bleikonzentration von $0,48 \pm 0,15 \mu \mathrm{mol} / \mathrm{l}(\overline{\mathrm{x}} \pm \mathrm{s})$.

Zur Beurteilung von Blut- und Harnbleikonzentrationen sei eine Empfehlung der "Conference on Inorganic Lead", Amsterdam 1968, aus einem Bericht von SCHLEGEL (16) wiedergegeben. Danach darf die berufliche Bleiaufnahme mit Sicherheit als akzeptabel bezeichnet werden, wenn die folgenden Werte nicht erreicht oder nicht überschritten werden: $3,38 \mu \mathrm{mol} / 1$ Blei im Vollblut und $0,63 \mu \mathrm{mol} / 1$ Blei im Harn. Weiterhin möchten wir aus den bisher erschienenen Angaben (17) über Bleikonzentrationen in Blut und Harn eine Tabelle hervorheben, die auch Grenzwerte für beruflich nicht bleibelastete Personen enthält (Tab. 2).

Tab. 2

Grenzwerte zur Beurteilung von Bleikonzentrationen in Blut und Harn

\begin{tabular}{lllll}
\hline & normal & akzeptabel & gesteigert & gefährlich \\
\hline $\mathrm{Pb}$ im Blut & & & & \\
$\begin{array}{c}{[\mu \mathrm{mol} / 1]} \\
\mathrm{Pb} \mathrm{im} \mathrm{Harn} \\
{[\mu \mathrm{mol} / 1]}\end{array}$ & $\leqq 1,93$ & $1,93-3,86$ & $3,86-5,8$ & $>5,8$ \\
\hline
\end{tabular}

Danach darf bei nicht bleigefährdeten Menschen ein Blutbleispiegel bis $1,93 \mu \mathrm{mol} / \mathrm{l}$, ein Harnbleispiegel bis $0,4 \mu \mathrm{mol} / 1$ als normal betrachtet werden.

\section{Diskussion}

KAHN et al. (9) hatte mit der Einführung der BootTechnik, bei der die Probe in einem $500 \mu \mathrm{l}$ fassenden Tantalschiffchen in die Flamme gebracht wird, beträchtliche Steigerungen der unteren Nachweisgrenze verschiedener leicht atomisierbarer Elemente wie Arsen, Blei, Cadmium, Quecksilber, Zink und einiger anderer erzielt. Blut oder Harn konnten zwar unvorbehandelt in das Boot pipettiert werden; doch ergab sich aus dem stets nur einzeln in unmittelbarer Nähe der Flamme durchführbaren Trocknungsvorgang ein erheblicher Zeitaufwand. Zudem sind Probenverluste während der Trocknung möglich, und Delves (8) wies auf die Abhängigkeit der Peakhöhen von der Lokalisation der Probe im Boot hin.

WhrTE (18) hingegen verwendete schon ein Absorptionsrohr, brachte jedoch die Probe mit Hilfe einer Platindrahtschlinge in die Flamme. Für Blutproben waren allerdings zuvor Veraschungen bei $450^{\circ} \mathrm{C}$ von 20 min Dauer erforderlich.
Die Probenbecher-Technik von Delves ist bestechend aufgrund ihres einfachen Prinzips. Dexves (8) verwandte bei seinem Verfahren das Absorptionsrohr und kleine Probentiegel, benutzte zur gleichzeitigen Trocknung mehrerer Proben eine Heizplatte und erzielte auf diese Weise neben hoher Probenfrequenz gute Ergebnisse. Der Vorzug seines Verfahrens liegt darin, daß die Verweildauer der Bleiatome im Strahlengang der $\mathrm{Pb}-$ Resonanzlinie erhöht wird, bevór sie das Rohr beidseits verlassen. Aus der längeren Verweildauer resultiert eine beträchtliche Steigerung der Empfindlichkeit und damit Verbesserung der Nachweisgrenze.

Die Validität seiner Probenbecher-Technik belegte Desves (8) durch einen Methodenvergleich. Er stellte 39 Werte dieses Verfahrens denjenigen einer von ihm automatisierten Dithizon-Methode gegenüber und ermittelte einen Korrelationskoeffizienten von $\mathrm{r}=0,989$ bei einer Steigerung der Regressionsgeraden von $y=1,10 x-7,36$. Ebenso ermittelten Joselow und BOGDEN (19) bei einem Vergleich der Mikroprobentechnik von DeLvËs mit dem Aspirationsverfahren (Probènzerstäubung) einen Körrelationskoeffizienten von über 0,9 .

Kerber und Fernandez (20) geben für die DelvesTechnik eine untere Nachweisgrenze für Blei in Lösungen von $1 \mathrm{ng} / \mathrm{ml}$ an. Das bedeutet gegenüber der konventionellen Atom-Absorptions-Spektrometrie eine Verbesserung um den Faktor 20. Als absolute Nachweisgrenze wurde 0,1 ng errechnet. Die gleiche Nachweisgrenze wird auch für Bleibestimmungen mit der Graphitrohrküvette angegeben.

Unsere Angaben zur Präzision in Serie für Bleibestimmungen im Blut sind für ähniliche Konzentrationen mit denen von Delves (8) vergleichbar. Auch für die Harnanalysen in Serie ergab sich ein davon nur gering abweichender Variationskoeffizient.

Die Präzision von Tag zu Tag brachte gegenüber den serienmäßigen Analysen niedrigere Werte der Variationskoeffizienten. Diese Verbesserung erklärt sich aus der täglich benutzten gleichen Kombination von nur 6 bzw. 12 Probentiegeln.

Ein Einfluß von Harnsubstanzen auf die BleiatomAbsorption konnte nicht festgestellt werden. Eine besondere Harnvorbereitung ist nicht erforderlich. Unumgänglich dagegen ist eine Probenvorbereitung für Harnbleianalysen mit der Graphitrohrküvette, worüber Döllefeld (21) berichtet. Bei diesem Verfahren konnten die organischen Harnbestandteile während der programmierbaren Veraschungsstufe nicht vollständig erschlossen werden, ohne daß es zu merklichen Bleiverlusten kam. Wie sich zeigte, ist Blei bei einer Veraschungstemperatur von $750^{\circ} \mathrm{C}$ flüchtig.

Die Hinweise im folgenden Abschnitt geben unsere Beobachtungen wieder. Besondere Sorgfalt erfordert die Montage und Justierung des Absorptionsrohrs hinsichtlich seiner Lage über dem Brenner und des optimalen Strahlendurchgangs. Das Rohr muß in seiner ganzen Länge über den Brennerschlitzen liegen. Ebenso 
ist das Anpassen des Probentiegels an die Probeneintrittsöffnung in Meßstellung exakt durchzuführen. Nur so ist einerseits eine gleichmäßige Temperatur in allen Abschnitten des Absorptionsrohrs sowie ein vollständiges Einströmen der Bleiatome in das Rohr gewährleistet. Nach Zünden der Flamme darf nicht versäumt werden, demineralisiertes Wasser über den Probenschlauch ansaugen zu lassen, andernfalls kommt es zur Uberhitzung des Brennerkopfes mit Deformierung und zum Abreißen der Flamme. Dies sind neben der Geräteinstellung wichtige Voraussetzungen für einen einwandfreien Ablauf des Meßvorgangs.

Zur Probenverarbeitung ist zu bemerken, daß die erste Trocknung der Blutprobe nicht ausgelassen werden darf. Die erste Trocknung reduziert die hohe Katalaseaktivität im Blut, so daß nach Zugabe von Perhydrol die Probe nur wenig aufschäumt und Probenverluste vermieden werden.

Die Probentiegel sind nicht, unbegrenzt lange haltbar. Nach mehrfachem Gebrauch treten bedingt durch die sich stets wiederholenden extremen Temperaturdifferenzen während der Messungen Absorptionseinbußen auf. Delves (8) fand unterhalb von 30 Messungen kein tiegelbedingtes Nachlassen der Absorption. Wie sich bei unseren Messungen zeigte, werden die Tiegel teilweise nach längerem Einsatz undicht und die $\mathrm{Ab}$ sorptionseinbußen sind dann auf Probenverluste zurückzuführen. Besonders schnell werden die Tiegel durch säurehaltige Proben unbrauchbar, so z. B. durch das Messen von Harnen, die zur Konservierung (u. a. mit Essigsäure) angesäuert wurden. Nach längerem Gebrauch sollte die Dichtigkeit der Tiegel öfters geprüft werden.

Auffallend sind bei der Probenbecher-Technik die deutlich niedrigeren Absorptionspeaks wäßriger Bleilösungen gegenüber denen von Blutproben gleichen Bleigehalts. Die Erklärung dafür sieht Delves (8) im Matrixeffekt des Blutes.

Abschließend sei auf die Frage nach der Wahl des Untersuchungsmaterials eingegangen, Blut oder Harn oder beide Materialien zur Kontrolle einer möglichen Bleibelastung zu verwenden. Nach LEHNERT (15) und vielen anderen Autoren ist die Bleibestimmung im Blut $\mathrm{zu}$ bevorzugen. Weniger geeignet erweist sich Serum, da etwa 95\% des Blutbleis in den Erythrocyten lagert. Harnbleibestimmungen sollten nur klinischstationär durchgeführt werden, weil unvollständiges Sammeln, Kontamination und Ablagerung von Bleisalzen im Harn die Bestimmung stören. Aber auch wegen der höheren Absorptionen bei Blutbleibestimmungen empfiehlt es sich, vorzugsweise Blutproben zu untersuchen. Harn für Bleibestimmungen sollte zwar angesäuert aufbewahrt werden, sofern er nicht unmittelbar verarbeitet werden kann, zur Messung ist er jedoch zu neutralisieren, um das zu schnelle Korrodieren der Tiegel zu verhüten. Zur. Aufbewahrung bleihaltiger Lösungen eignen sich besonders gut Gefäße aus Polyäthylen wegen seiner unpolaren Eigenschaft.

\section{Literatur}

1. WAChtER, H. \& Sallaberger, G. (1971), Wien. Klin. Wochenschr. 83, 869-876. - 2. Christina, G. D. (1972), Deut. Ges. Klin. Chem., Mitt. Nr. 3-5, 47-84. - 3. Berman, Eleanor (1964), At. Absorption Newslett. 3, 111-114. - 4. WirLIs, J. B. (1965), Clin. Chem. 11, 251-258. - 5. Sprague, Sabina \& Slavin, W. (1966), At. Absorption Newslett. 5, 9-10. - 6 . LeHNert, G. \& Schaller, K. H. (1967), Med. Welt 18, 1131 bis 1133. - 7. Lẹhnert, G., Schaller, K. H. \& Szadkowski, D. (1969), diese Z. 7, 310. - 8. Delves, H. T. (1970), Analyst 95, 431-438. - 9. Kahi, H. L., Peterson, G. E. \& Schallis, J. E, (1969), Analysentechn. Berichte Nr. 17, Bodenseewerk PerkinElmer, Uberlingen. - 10. Matousex, J. P. \& Stevens, J. (1971). Clin. Chem. 17, 363-368. - 11. Allan, J. E. (1961), Spectrochim. Acta 17, 459-466. - 12. HerrmanN, R. (1965), diese Z.
3, 178-186. - 13. Willis, J. B. (1960), Spectrochim. Acta 16 259-272. - 14. Paschen, K. \& SpiekermanN, P. G. (1970), Deut. Med. Wochenschr. 51, 2577-2581. - 15. LeHnert, G. (1968), Schriftenreihe Arbeitsmed., Sozialmed., Arbeitshyg. Nr. 21, Gentner Verlag, Stuttgart. - 16. Schlegel, H. (1969), Arbeitsmed., Sozialmed., Arbeitshyg 2,.56-57. -17. Diagnosis of Inorganic Lead Poisoning. A Statement (1968), Brit. Med. J. 501. -- 18. White, R. A., „zit. nach Fernandez, F. J. \& KaHN, H. I.. (1971), At. Absorption Newslett. 10, 1-5“. - 19. Joselow, M. M. \& BogdEN, J. D. (1972), At. Absorption Newslett. 11, 12i-128. - 20. Kerber, J. D. \& Fernandez, F. J. (1971), At. Absorption Newslett. 10, 78-80. - 21. Döllefeld, E. (1971), Arztl. Lab. 17, 369-371.

Dr. G. Heinemann

Institut für Klinische Chemie und Klinische Biochemie Klinikum Steglitz der FU Berlin

1 Berlin 45

Hindenburgdamm 30 